\title{
Effects of 6-week squat, deadlift, or hip thrust training program on speed, power, agility, and strength in experienced lifters: A pilot study
}

\author{
Michael B. Zweifel, Andrew D. Vigotsky, Bret Contreras, Wycliffe W. Njororai Simiyu
}

Objectives: To compare the ergogenic effects of back squats, deadlifts, and hip thrusts.

Design: Pilot randomized-controlled trial

Methods: In order to determine the feasibility of such a large training study, a pilot study was carried out with 26 male and female participants $($ age $=22.15 \pm 2.2$ years; height $=180.17 \pm 8.37 \mathrm{~cm}$; body mass $=87.27 \pm 15.72 \mathrm{~kg})$. Subjects performed squats $(n=8)$, hip thrusts $(n=8)$, deadlifts $(n=6)$, or nothing (control) $(n=4)$ for three training sessions a week, for six weeks; thereafter, measures of sprinting performance, vertical jump, broad jump, strength, and change of direction were compared to baseline.

Results: This pilot study was carried out successfully. Effect-sizes, medians, and interquartile ranges for all possible comparisons have been presented for power analyses.

Conclusion: Although all of the studied interventions show promise, larger investigations are necessary in order to draw more definitive, applicable conclusions.

(Journal of Trainology 2017;6:13-17)

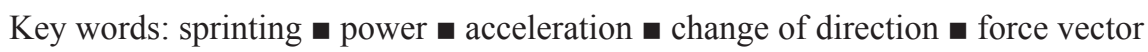

\section{INTRODUCTION}

Two exercises that are commonly utilized to increase lower body strength are the back squat and deadlift. The back squat is regarded as one of the most effective exercises used to enhance athletic performance; a breadth of literature exists elucidating the ergogenic effects of the back squat ${ }^{1,2}$, including one meta-analysis that found a correlation between back squat strength and sprinting speed ${ }^{3}$. The deadlift is also regarded as an important lower body exercise for improving strength and power ${ }^{4}$, and there is still literature to support its use ${ }^{5,6}$.

It has been shown that it takes exceptionally large increases in 1RM back squat strength ( 9-44\%) to only slightly increase sprinting speed $(1-4 \%))^{7,8}$ The squat and deadlift are primarily sagittal plane movements with emphases on axial (top-down) loading. ${ }^{9}$ Many movements in sport are multiplanar in nature. For enhanced sport performance, specificity is an important aspect of training, and the back squat and deadlift lack specificity of anteroposterior, lateral, and transverse force application. ${ }^{9}$

An exercise that is gaining popularity for the enhancement of sport performance is the barbell hip thrust ${ }^{10}$. The hip thrust differs from the back squat and deadlift in that it is loaded anteroposteriorly rather than axially. Recent research has shown high levels of horizontal force application is related to faster sprinting speeds. ${ }^{11-15}$ Contreras and colleagues ${ }^{16}$ found that the hip thrust is effective for improving both horizontal jump distance and sprinting performance.

The purpose of this study was to determine the plausibility and collect preliminary effect sizes for a study that investigates the effects of a 6-week resistance-training program of back squats, deadlifts, or hip thrusts on performances in the 10-yard dash, 40-yard dash, countermovement jump, broad jump, and estimated 1RM in trained subjects.

\section{Subjects}

\section{METHODS}

Twenty-six participants with an athletic background were recruited to participate in this study (age $=22.15 \pm 2.2$ years; height $=180.17 \pm 8.37 \mathrm{~cm}$; body mass $=87.27 \pm 15.72 \mathrm{~kg}$ ) (Table 1). The participants were divided into four groups, consisting of the back squat $(n=8)$, deadlift $(n=6)$, hip thrust $(n=8)$, or control $(n=4)$ groups. All participants had at least one year of resistance training experience, including experience in all three of the experimental exercises. Before beginning, all subjects agreed to and signed an informed consent. Participants were asked to refrain from any exercise outside of the study. This study was approved by the Institutional Review Board at the University of Texas at Tyler.

\section{Procedures}

After signing the informed consent form, subjects were randomly (simple randomization) placed into their respective

Received November 13, 2016; accepted February 11, 2017

From the Building Better Athletes, LLC (M.B.Z.), Leon Root, M.D. Motion Analysis Laboratory, Hospital for Special Surgery, New York, NY (A.D.V.), BC Athletics, LLC., Phoenix, Arizona (B.C.), and Department of Health and Kinesiology, University of Texas at Tyler (W.W.N.S.)

Communicated by Takashi Abe, PhD

Correspondence to Michael Zweifel, 3188 Hughes Ct., Dubuque, IA 52003, USA.

E-mail: buildingbetterathletes.bba@gmail.com

Journal of Trainology 2017;6:13-17 @2012 The Active Aging Research Center

http://trainology.org/ 
Table 1 Participant Details (During Pre-Testing)

\begin{tabular}{cccccc}
\hline & Overall & Squat Group & Deadlift Group & Hip Thrust Group & Control Group \\
\hline Height (cm) & $180.17 \pm 8.37$ & $181.37 \pm 9.24$ & $182.99 \pm 5.91$ & $178.20 \pm 8.76$ & $177.48 \pm 10.39$ \\
\hline Weight (kg) & $86.75 \pm 15.91$ & $92.55 \pm 18.48$ & $86.22 \pm 15.20$ & $82.57 \pm 17.71$ & $84.32 \pm 7.15$ \\
\hline Age (years) & $22.15 \pm 2.20$ & $22.25 \pm 2.25$ & $21.17 \pm 2.71$ & $22.38 \pm 2.07$ & $23.00 \pm 1.83$ \\
\hline CMJ (cm) & $61.30 \pm 10.84$ & $61.40 \pm 9.79$ & $64.22 \pm 10.61$ & $57.37 \pm 10.73$ & $64.45 \pm 15.08$ \\
\hline Broad Jump (cm) & $235.68 \pm 30.59$ & $237.17 \pm 29.87$ & $244.26 \pm 26.02$ & $226.70 \pm 22.09$ & $237.81 \pm 55.43$ \\
\hline 10-Yard (sec) & $1.89 \pm 0.14$ & $1.87 \pm 0.13$ & $1.87 \pm 0.17$ & $1.92 \pm 0.13$ & $1.87 \pm 0.21$ \\
\hline 40-Yard (sec) & $5.38 \pm 0.41$ & $5.33 \pm 0.32$ & $5.28 \pm 0.44$ & $5.45 \pm 0.40$ & $5.47 \pm 0.66$ \\
\hline 5-10-5 (sec) & $4.91 \pm 0.41$ & $4.86 \pm 0.40$ & $4.86 \pm 0.37$ & $4.98 \pm 0.38$ & $4.97 \pm 0.66$ \\
\hline EST 1RM Squat (kg) & $125.35 \pm 41.26$ & $136.93 \pm 30.82$ & $124.24 \pm 44.85$ & $111.08 \pm 38.04$ & $132.39 \pm 65.38$ \\
\hline EST 1RM Deadlift (kg) & $142.31 \pm 39.88$ & $157.95 \pm 30.42$ & $142.43 \pm 42.87$ & $127.27 \pm 42.85$ & $140.91 \pm 49.86$ \\
\hline EST 1RM Hip Thrust (kg) & $145.80 \pm 33.51$ & $156.82 \pm 27.06$ & $140.30 \pm 35.51$ & $144.89 \pm 34.61$ & $133.85 \pm 46.62$ \\
\hline
\end{tabular}

(CMJ = Countermovement Jump; RM= Repetition Maximum)

groups. Next, pre-tests were performed to evaluate subjects' acceleration in the 10-yard sprint; top-end sprinting speed in the 40-yard sprint; jumping performance in the countermovement and broad jumps; change of direction performance in the pro agility test (5-10-5); and a $3 \mathrm{RM}$ in the back squat, deadlift, and hip thrust to estimate 1RM maximal strength. The study involved two days of testing, used for both pre- and post-tests, as described hereinafter.

\section{Dynamic Warm-up}

Before testing and each training session thereafter, subjects first went through a dynamic warm-up, consisting of 15 yards of each of the following dynamic stretches: knee hugs, walking lunges, lateral lunges, "A" skips, "A" runs, cariocas, backward open-hips, zombie walks, and finally, "A" runs again.

\section{Countermovement Jump}

Countermovement jump was tested using the Just Jump Mat (Probiotics, Huntsville, AL). The Just Jump Mat calculates vertical jump height by recording the time that an individual is off the ground - that is, between takeoff and landing - during the vertical jump. Subjects were instructed to jump vertically, as high as possible, and land in an athletic position.

\section{Sprinting Time}

Ten- and 40-yard dash times were evaluated using the Speed Trap I Timer (Power Systems, Inc., Knoxville, TN), which is an electronic timing system with a precision of 10 milliseconds. Each subject started in a three-point stance of their choosing. The timer was started when each subject's thumb left the thumb pad timer, and the timer was stopped using a laser sensor.

\section{Broad Jump}

Broad jump was evaluated using a standard measuring tape. Subjects were instructed to jump as far as possible and were required to stick the landing and stabilize in order for a mea- surement to be taken. Measurements were taken from the starting line (toes) to the heel closest to the starting line.

\section{Pro Agility Test}

The Pro Agility (5-10-5) test was evaluated using the Coach's Eye (TechSmith, Okemos, Michigan). Each subject's Pro Agility trial was replayed in Coach's Eye and was evaluated frame-by-frame, from the first movement to when the subject's chest crossed the finish line.

\section{RM Testing}

Subjects started with a relatively light load ( $\sim 50 \% 1 \mathrm{RM})$ and increased weight progressively, at their preferred rate, until the coach (MZ) deemed the weight to be an appropriate 3RM. Loads were adjusted after each trial according to how each subject felt after completing a repetition, or depending upon what the coach deemed would be an appropriate change in load. Using the 3RM loads, $1 \mathrm{RM}$ was estimated using the tables provided by Baechle and Earle ${ }^{17}$. The procedures for each lift are summarized herein.

\section{Back Squat}

Three-repetition maximum of the back squat was tested with a depth of parallel, wherein the subject descended until his or her femur was parallel with the ground and in a high bar position. For each squat, the subject's stance width was approximately shoulder width with the feet flared out to each subject's level of comfort.

\section{Hip Thrust}

Forty-eight hours of rest was provided following the first testing day, after which subjects returned for hip thrust 3RM testing, which was performed in accordance with Contreras and colleagues ${ }^{10}$. Specifically, a barbell with bumper plates rested atop the subject's hip crease while their mid-to-upper back was set on a bench. The subject was instructed to extend his or her hips, pushing through their heels, until they were 
Table 2 Training Program (All experimental groups performed this program with their specific lift)

\begin{tabular}{|c|c|c|c|c|c|c|c|c|c|}
\hline & \multicolumn{3}{|c|}{ Day 1} & \multicolumn{3}{|c|}{ Day 2} & \multicolumn{3}{|c|}{ Day 3} \\
\hline & Set $x$ Reps & Load (\%) & Rest (min) & Set $\mathrm{x}$ Reps & Load (\%) & Rest (min) & Set x Reps & Load (\%) & Rest (min) \\
\hline \multirow{4}{*}{ Week 1} & $1 \times 5$ & 30 & 1.5 & $1 \times 5$ & 30 & 1.5 & $1 \times 5$ & 30 & 1.5 \\
\hline & $1 \times 5$ & 50 & 1.5 & $1 \times 5$ & 50 & 1.5 & $1 \times 5$ & 50 & 1.5 \\
\hline & $4 \times 8$ & 72 & 1.5 & $4 \times 6$ & 77 & 1.5 & $4 \times 8$ & 80 & 1.5 \\
\hline & & & & & & & $1 \times$ AMRAP & 70 & \\
\hline \multirow{4}{*}{ Week 2} & $1 \times 5$ & 30 & 1.5 & $1 \times 5$ & 30 & 1.5 & $1 \times 5$ & 30 & 1.5 \\
\hline & $1 \times 5$ & 50 & 1.5 & $1 \times 5$ & 50 & 1.5 & $1 \times 5$ & 50 & 1.5 \\
\hline & $4 \times 10$ & 72 & 1.5 & $4 \times 8$ & 77 & 1.5 & $4 \times 6$ & 80 & 1.5 \\
\hline & & & & & & & $1 \times$ AMRAP & 70 & \\
\hline \multirow{4}{*}{ Week 3} & $1 \times 5$ & 30 & 1.5 & $1 \times 5$ & 30 & 1.5 & $1 \times 5$ & 30 & 1.5 \\
\hline & $1 \times 5$ & 50 & 1.5 & $1 \times 5$ & 50 & 1.5 & $1 \times 5$ & 50 & 1.5 \\
\hline & $4 \times 4$ & 85 & 4 & $4 \times 5$ & 80 & 4 & $2 \times 5$ & 82 & 4 \\
\hline & & & & & & & $2 \times 2$ & 90 & 4 \\
\hline \multirow{4}{*}{ Week 4} & $1 \times 5$ & 30 & 1.5 & $1 \times 5$ & 30 & 1.5 & $1 \times 5$ & 30 & 1.5 \\
\hline & $1 \times 5$ & 50 & 1.5 & $1 \times 5$ & 50 & 1.5 & $1 \times 5$ & 50 & 1.5 \\
\hline & $5 \times 3$ & 88 & 4 & $5 \times 4$ & 82 & 4 & $2 \times 2$ & 95 & 4 \\
\hline & & & & & & & $2 \times 1$ & 100 & 4 \\
\hline \multirow{3}{*}{ Week 5} & $1 \times 5$ & 30 & 1.5 & $1 \times 5$ & 30 & 1.5 & $1 \times 5$ & 30 & 1.5 \\
\hline & $1 \times 5$ & 50 & 1.5 & $1 \times 5$ & 50 & 1.5 & $1 \times 5$ & 50 & 1.5 \\
\hline & $5 \times 2$ & 50 & 2.5 & $5 \times 4$ & 25 & 2.5 & $5 \times 3$ & 35 & 2.5 \\
\hline \multirow{3}{*}{ Week 6} & $1 \times 5$ & 30 & 1.5 & $1 \times 5$ & 30 & 1.5 & $1 \times 5$ & 30 & 1.5 \\
\hline & $1 \times 5$ & 50 & 1.5 & $1 \times 5$ & 50 & 1.5 & $1 \times 5$ & 50 & 1.5 \\
\hline & $6 \times 2$ & 55 & 2.5 & $6 \times 4$ & 30 & 2.5 & $6 \times 3$ & 40 & 2.5 \\
\hline
\end{tabular}

AMRAP = as many repetitions as possible; Reps $=$ repetitions

fully extended. If a subject could not fully extend his or her hips with a given load, the repetition was not counted.

\section{Deadlift}

Subjects' 3RM deadlift was tested following hip thrust testing. The deadlift was to be pulled in the 'conventional' position off the floor ${ }^{4}$. Subjects were allowed to "touch and go" or reset after each repetition, depending on their preference.

\section{Training Protocol}

After pre-testing, participants were provided 72-hours of rest before beginning the six-week training (or control) period. During these 6-weeks, participants were asked to reduce and avoid as much conflicting activity as possible. Subjects trained three days per week with total emphasis on their singular lift. There was 48-hours of rest after the first two training sessions of the week and a 72-hour rest following the third training session of the week (Trained on Monday, Wednesday, Friday). Training followed a condensed linear periodization model consisting of a two-week emphasis on hypertrophy, two-week emphasis on strength, and a two-week emphasis on power. Thus, a total of six training sessions were used for each block - hypertrophy, strength, and power - for a total of 18 training sessions. The specific training protocol that each experimental group executed can be found in Table 2. At the end of the six weeks of training, subjects re-tested the same aforementioned performance tests with the same methodology described previously.

\section{Statistical Analysis}

Because this was intended to be a pilot study, inferential statistics were not calculated. However, effect-sizes and descriptive statistics were calculated and reported so that future studies may use these data for sample size estimates. Due to the small sample size, non-parametric tests were used to obtain z-scores, from which effect-sizes were calculated. All effectsizes were calculated using the formula $r=\frac{z}{\sqrt{N}}$, where $\mathrm{z}$ is the z-score obtained from a Wilcoxon signed-rank test or Wilcoxon rank-sum test, for within- and between-group comparisons, respectively. Between-group comparisons were performed between each group, for a total of 6 comparisons: squat vs. hip thrust; squat vs. deadlift; squat vs. control; hip thrust vs. deadlift; hip thrust vs. control; and deadlift vs. control. In addition, medians and interquartile of pre measures, post measures, and changes were reported to allow the reader perform further analyses, if desired.

Pearson correlation coefficients were calculated and report- 
ed in a correlation matrix between all outcome (post-training interventions) measures, including countermovement jump, broad jump, 10-yard dash, 40-yard dash, the Pro Agility test, relative squat strength, relative deadlift strength, and relative hip thrust strength.

\section{RESULTS}

Medians, interquartile ranges, and effect-sizes for strength and performance outcomes can be found in Table 3. Moreover, correlation coefficients observed between different performance outcomes are presented in Table 4.

\section{DISCUSSION}

This pilot study successfully demonstrated that an investigation between the squat, deadlift, and hip thrust is feasible; in addition, this present study provides effect-sizes from which

Table 3 Average pre, post, delta, and effect-sizes within and between all groups.

\begin{tabular}{|c|c|c|c|c|c|c|c|c|c|}
\hline \multirow[b]{2}{*}{ Outcome } & \multirow[b]{2}{*}{ Group } & \multirow{2}{*}{$\begin{array}{l}\text { Median Pre } \\
(\mathrm{Q} 1, \mathrm{Q} 3)\end{array}$} & \multirow{2}{*}{$\begin{array}{c}\text { Median Post } \\
(\mathrm{Q} 1, \mathrm{Q} 3)\end{array}$} & \multirow{2}{*}{$\begin{array}{l}\text { Median Delta } \\
(\mathrm{Q} 1, \mathrm{Q} 3)\end{array}$} & \multicolumn{5}{|c|}{ Pearson's $r$} \\
\hline & & & & & $\begin{array}{l}\text { Within- } \\
\text { group }\end{array}$ & $\begin{array}{l}\text { vs. hip } \\
\text { thrust }\end{array}$ & $\begin{array}{c}\text { vs. } \\
\text { squat }\end{array}$ & $\begin{array}{c}\text { vs. } \\
\text { deadlift }\end{array}$ & $\begin{array}{c}\text { vs. } \\
\text { control }\end{array}$ \\
\hline \multirow{4}{*}{$\begin{array}{l}\text { Vertical } \\
\text { Jump } \\
(\mathrm{cm})\end{array}$} & Hip Thrust & $57.53(54.54,61.85)$ & $60.20(54.95,63.12)$ & $1.79(1.32,2.29)$ & 0.60 & & 0.18 & 0.09 & 0.29 \\
\hline & Squat & $61.60(55.37,64.01)$ & $61.60(53.72,67.88)$ & $-1.27(-2.60,4.07)$ & 0.12 & -0.18 & & -0.28 & -0.15 \\
\hline & Deadlift & $69.98(61.22,70.74)$ & $70.99(60.52,71.57)$ & $1.14(0.63,2.61)$ & 0.43 & -0.09 & 0.28 & & 0.07 \\
\hline & Control & $71.12(63.63,71.94)$ & $73.03(64.52,73.79)$ & $1.02(0.13,1.71)$ & 0.39 & -0.29 & 0.15 & -0.07 & \\
\hline \multirow{4}{*}{$\begin{array}{l}\text { Broad } \\
\text { Jump } \\
(\mathrm{cm})\end{array}$} & Hip Thrust & $223.52(214.63,233.68)$ & $233.68(222.25,250.19)$ & $7.62(7.62,14.1)$ & 0.63 & & 0.36 & 0.09 & 0.52 \\
\hline & Squat & $243.84(212.09,256.54)$ & $248.92(218.44,266.07)$ & $5.08(-6.35,8.26)$ & 0.14 & -0.36 & & -0.31 & 0.10 \\
\hline & Deadlift & $250.19(242.57,255.91)$ & $262.89(257.18,266.70)$ & $8.89(3.18,14.61)$ & 0.61 & -0.09 & 0.31 & & 0.37 \\
\hline & Control & $257.81(229.55,266.07)$ & $257.41(231.80,265.11)$ & $3.18(-1.79,6.67)$ & 0.20 & -0.52 & -0.10 & -0.37 & \\
\hline \multirow{4}{*}{$\begin{array}{c}\text { 10-yard } \\
\text { Dash } \\
(\mathrm{sec})\end{array}$} & Hip Thrust & $1.93(1.87,1.99)$ & $1.90(1.84,1.98)$ & $-0.01(-0.03,0.00)$ & -0.55 & & -0.27 & -0.23 & -0.15 \\
\hline & Squat & $1.86(1.78,1.95)$ & $1.89(1.76,1.97)$ & $-0.01(-0.02,0.04)$ & 0.04 & 0.27 & & 0.16 & 0.02 \\
\hline & Deadlift & $1.80(1.76,1.92)$ & $1.80(1.75,1.95)$ & $-0.01(-0.01,0.01)$ & -0.19 & 0.23 & -0.16 & & -0.07 \\
\hline & Control & $1.78(1.75,1.90)$ & $1.83(1.76,1.96)$ & $0.00(-0.04,0.06)$ & 0.07 & 0.15 & -0.02 & 0.07 & \\
\hline \multirow{4}{*}{$\begin{array}{c}\text { 40-yard } \\
\text { Dash } \\
(\mathrm{sec})\end{array}$} & Hip Thrust & $5.40(5.35,5.53)$ & $5.31(5.27,5.40)$ & $-0.09(-0.10,-0.07)$ & -0.63 & & -0.34 & -0.31 & -0.54 \\
\hline & Squat & $5.29(5.11,5.63)$ & $5.24(5.06,5.51)$ & $-0.06(-0.07,-0.01)$ & -0.28 & 0.34 & & 0.02 & -0.15 \\
\hline & Deadlift & $5.14(5.01,5.28)$ & $5.18(4.97,5.29)$ & $-0.04(-0.09,-0.02)$ & -0.30 & 0.31 & -0.02 & & -0.20 \\
\hline & Control & $5.33(5.05,5.75)$ & $5.37(4.98,5.87)$ & $-0.03(-0.05,0.03)$ & -0.13 & 0.54 & 0.15 & 0.20 & \\
\hline \multirow{4}{*}{$\begin{array}{c}\text { Pro } \\
\text { Agility } \\
(5-10-5) \\
(\mathrm{sec})\end{array}$} & Hip Thrust & $5.00(4.87,5.14)$ & $4.86(4.61,5.09)$ & $-0.07(-0.14,-0.04)$ & -0.58 & & 0.20 & -0.10 & 0.02 \\
\hline & Squat & $4.81(4.66,4.97)$ & $4.69(4.57,4.89)$ & $-0.13(-0.31,-0.06)$ & -0.42 & -0.20 & & -0.17 & -0.29 \\
\hline & Deadlift & $4.71(4.68,4.95)$ & $4.71(4.60,4.77)$ & $-0.04(-0.22,0.06)$ & -0.21 & 0.10 & 0.17 & & 0.00 \\
\hline & Control & $4.81(4.54,5.23)$ & $4.78(4.51,5.17)$ & $-0.06(-0.10,-0.03)$ & -0.65 & -0.02 & 0.29 & 0.00 & \\
\hline \multirow{4}{*}{$\begin{array}{l}\text { Squat } \\
(\mathrm{kg})\end{array}$} & Hip Thrust & $95.46(83.52,138.64)$ & $97.73(87.50,144.32)$ & $4.55(0.00,5.69)$ & 0.32 & & -0.57 & 0.30 & 0.15 \\
\hline & Squat & $131.82(120.45,162.50)$ & $150.00(123.86,182.96)$ & $12.50(7.96,20.50)$ & 0.62 & 0.57 & & 0.65 & 0.52 \\
\hline & Deadlift & $136.37(112.50,153.41)$ & $131.82(112.50,147.73)$ & $0.00(-3.41,0.00)$ & -0.10 & -0.30 & -0.65 & & -0.18 \\
\hline & Control & $138.64(105.68,165.34)$ & $138.64(104.55,169.32)$ & $0.00(-1.14,3.98)$ & 0.07 & -0.15 & -0.52 & 0.18 & \\
\hline \multirow{4}{*}{$\begin{array}{c}\text { Deadlift } \\
(\mathrm{kg})\end{array}$} & Hip Thrust & $118.18(88.64,150.00)$ & $129.55(100.00,154.55)$ & $9.09(4.55,13.64)$ & 0.59 & & 0.51 & -0.07 & 0.37 \\
\hline & Squat & $161.37(151.14,173.86)$ & $161.37(151.14,180.69)$ & $0.00(0.00,1.14)$ & 0.16 & -0.51 & & -0.46 & -0.22 \\
\hline & Deadlift & $152.28(130.68,170.46)$ & $161.37(143.19,176.14)$ & $9.09(5.68,12.50)$ & 0.55 & 0.07 & 0.46 & & 0.41 \\
\hline & Control & $154.55(125.00,170.46)$ & $154.55(125.91,172.73)$ & $1.82(0.00,5.00)$ & 0.50 & -0.37 & 0.22 & -0.41 & \\
\hline \multirow{4}{*}{$\begin{array}{l}\text { Hip } \\
\text { Thrust } \\
\text { (kg) }\end{array}$} & Hip Thrust & $143.19(119.32,155.68)$ & $159.09(129.55,179.54)$ & $22.73(11.37,27.27)$ & 0.62 & & 0.57 & 0.35 & 0.72 \\
\hline & Squat & $165.91(143.18,169.32)$ & $161.37(153.41,184.09)$ & $0.00(0.00,6.82)$ & 0.27 & -0.57 & & -0.28 & 0.38 \\
\hline & Deadlift & $150.00(140.91,159.09)$ & $154.55(125.91,172.73)$ & $11.68(5.85,13.64)$ & 0.58 & -0.35 & 0.28 & & 0.62 \\
\hline & Control & $138.64(112.26,160.23)$ & $138.64(109.09,160.23)$ & $0.00(-3.17,0.00)$ & -0.35 & -0.72 & -0.38 & -0.62 & \\
\hline
\end{tabular}

The skew-symmetric nature of the between-group effect-size matrices should be interpreted such that a positive value indicates a greater increase or smaller decrease for the row relative to the column. $Q 1=$ first quartile; $Q 3=$ third quartile 
Table 4 Correlation Coefficients - Performance Tests (Based On Post-Training Results)

\begin{tabular}{|c|c|c|c|c|c|c|c|c|c|c|}
\hline Variable & Hgt & $W g t$ & CMJ & Broad & $10 y$ & $40 y$ & $5-10-5$ & $R-S q t$ & $R-D L$ & $R-H P$ \\
\hline Height & 1 & & & & & & & & & \\
\hline Weight & 0.47 & 1 & & & & & & & & \\
\hline CMJ & 0.12 & 0.05 & 1 & & & & & & & \\
\hline Broad & 0.13 & 0.23 & 0.89 & 1 & & & & & & \\
\hline $10 y$ & -0.14 & 0.00 & -0.88 & -0.84 & 1 & & & & & \\
\hline $40 y$ & -0.06 & 0.07 & -0.81 & -0.78 & 0.91 & 1 & & & & \\
\hline $5-10-5$ & -0.16 & -0.11 & -0.84 & -0.82 & 0.89 & 0.93 & 1 & & & \\
\hline$R-S q t$ & -0.20 & -0.05 & 0.75 & 0.69 & -0.72 & -0.75 & -0.72 & 1 & & \\
\hline$R-D L$ & -0.07 & -0.17 & 0.78 & 0.75 & -0.85 & -0.84 & -0.78 & 0.88 & 1 & \\
\hline$R-H P$ & -0.17 & -0.28 & 0.56 & 0.55 & -0.70 & -0.81 & -0.68 & 0.71 & 0.77 & 1 \\
\hline
\end{tabular}

(Broad = Broad Jump; CMJ=Countermovement JumpR-DL = Relative Deadlift Strength; R-HP = Relative Hip Thrust Strength; ; $R-S q t=$ Relative Squat Strength)

researchers can calculate appropriate sample sizes. One must be cautious as not to make conclusions from these data, as this study was underpowered. While other studies suggest that different exercises transfer to one another ${ }^{16,18}$, such a conclusion cannot be directly drawn from pilot data.

It is recommended that researchers not only utilize these effect-sizes for power analyses, but also for hypothesis generation and trial design. What is often considered to be large effect-sizes $(r \geq 0.5)$ were observed for three outcomes: hip thrusts vs. control on broad jumps $(r=0.52)$, hip thrusts vs. control on 40-yard sprint time $(r=-0.54)$, and deadlifts vs. control on hip thrusts $(r=0.62)$; therefore, it is likely that these relationships are strongest and will be easiest to carry out a well-powered study on. Of particular interest are the first two outcomes, broad jump and 40-yard sprint time, as these measures are representative of an ergogenic effect and transference. Training interactions may also be important to take into account in future studies, especially for athletes. Outcomes from one exercise may have interactive effects, linear or nonlinear, with other training interventions, exercises, or drills, such that outcomes are not always predictable. As such, the results from this pilot study are only relevant for the protocol and population employed by this study, and the reported effect-sizes cannot be extrapolated to other programs or populations.

\section{CONCLUSION}

This study was strictly a feasibility study; the observed outcomes should not be extrapolated or applied, and no firm conclusions can be drawn.

\section{REFERENCES}

1. McBride JM, Blow D, Kirby TJ, Haines TL, Dayne AM, Triplett NT. Relationship between maximal squat strength and five, ten, and forty yard sprint times. J Strength Cond Res 2009;23:1633-1636.

2. Comfort P, Haigh A, Matthews MJ. Are changes in maximal squat strength during preseason training reflected in changes in sprint performance in rugby league players? J Strength Cond Res 2012;26:772-776.

3. Seitz LB, Reyes A, Tran TT, Saez de Villarreal E, Haff GG. Increases in lower-body strength transfer positively to sprint performance: a systematic review with meta-analysis. Sports Med 2014;44:1693-1702.

4. Bird S, Barrington-Higgs B. Exploring the deadlift. Strength Cond J 2010;32:46-51.

5. Swinton PA, Lloyd R, Keogh JW, Agouris I, Stewart AD. Regression models of sprint, vertical jump, and change of direction performance. $J$ Strength Cond Res 2014;28:1839-1848.

6. Thompson BJ, Stock MS, Shields JE, et al. Barbell deadlift training increases the rate of torque development and vertical jump performance in novices. J Strength Cond Res 2015;29:1-10.

7. Cronin J, Ogden T, Lawton T. Does increasing maximal strength improve sprint running performance? Strength Cond J 2007;29:86-95.

8. Jacobson BH, Conchola EG, Glass RG, Thompson BJ. Longitudinal morphological and performance profiles for American, NCAA Division I football players. J Strength Cond Res 2013;27:2347-2354.

9. Young WB. Transfer of strength and power training to sports performance. Int J Sports Physiol Perform 2006;1:74-83.

10. Contreras B, Cronin J, Schoenfeld B. Barbell hip thrust. Strength Cond J 2011;33:58-61.

11. Brughelli M, Cronin J, Chaouachi A. Effects of running velocity on running kinetics and kinematics. J Strength Cond Res 2011;25:933-939.

12. Kawamori N, Nosaka K, Newton RU. Relationships between ground reaction impulse and sprint acceleration performance in team sport athletes. J Strength Cond Res 2013;27:568-573.

13. Morin JB, Edouard P, Samozino P. Technical ability of force application as a determinant factor of sprint performance. Med Sci Sports Exerc 2011;43:1680-1688.

14. Morin JB, Slawinski J, Dorel S, et al. Acceleration capability in elite sprinters and ground impulse: Push more, brake less? J Biomech 2015;48:3149-3154.

15. Morin JB, Bourdin M, Edouard P, Peyrot N, Samozino P, Lacour JR. Mechanical determinants of 100-m sprint running performance. Eur J Appl Physiol 2012;112:3921-3930.

16. Contreras B, Vigotsky AD, Schoenfeld BJ, et al. Effects of a six-week hip thrust versus front squat resistance training program on performance in adolescent males: A randomized-controlled trial. J Strength Cond Res 2016 [Epub ahead of print].

17. Baechle TR, Earle RW. Essentials of strength training and conditioning. 3rd ed. Champaign, IL: Human Kinetics; 2008.

18. Speirs DE, Bennett M, Finn CV, Turner AP. Unilateral vs Bilateral Squat training for Strength, Sprints and Agility in Academy Rugby Players. $J$ Strength Cond Res 2016;30:386-392. 